\title{
.
}

Revista Educación

ISSN: 0379-7082

ISSN: 2215-2644

revedu@gmail.com

Universidad de Costa Rica

Costa Rica

\section{Motivación hacia las matemáticas de estudiantes de bachillerato de modalidad mixta y presencial}

Jiménez Villalpando, Amaranta; Garza Kanagusiko, Arianna; Méndez Flores, Cindy Patricia; Mendoza Carrillo, Jesús; Acevedo Mendoza, Jorge; Arredondo Contreras, Luis Carlos; Quiroz Rivera, Samantha Motivación hacia las matemáticas de estudiantes de bachillerato de modalidad mixta y presencial

Revista Educación, vol. 44, núm. 1, 2020

Universidad de Costa Rica, Costa Rica

Disponible en: http://www.redalyc.org/articulo.oa?id=44060092014

DOI: https://doi.org/10.15517/revedu.v44i1.35282

Esta obra está bajo una Licencia Creative Commons Atribución-NoComercial-SinDerivar 3.0 Internacional. 


\section{Motivación hacia las matemáticas de estudiantes de bachillerato de modalidad mixta} y presencial

Student Motivation towards Mathematics at Blended Learning and Place-Based Classroom High Schools

Amaranta Jiménez Villalpando

Universidad Autónoma de Coabuila, México

amaranta.jimenez@hotmail.com

(iD) http://orcid.org/0000-0003-3747-0500

Arianna Garza Kanagusiko

Universidad Autónoma de Coabuila, México

ariannagarzak@hotmail.com

(iD) http://orcid.org/0000-0003-2822-5570

Cindy Patricia Méndez Flores

Universidad Autónoma de Coahuila, México

patmendez31@gmail.com

iD http://orcid.org/0000-0002-4753-7601

Jesús Mendoza Carrillo

Universidad Autónoma de Coabuila, México

jemendozac@uadec.edu.mx

iD http://orcid.org/0000-0003-0213-5243

Jorge Acevedo Mendoza

Universidad Autónoma de Coabuila, México

jorge_acevedo96@yahoo.com.mx

(iD http://orcid.org/0000-0001-8317-2980

Luis Carlos Arredondo Contreras

Universidad Autónoma de Coabuila, México

lic.luiscarlosarredondo@gmail.com

iD http://orcid.org/0000-0002-2195-0887

Samantha Quiroz Rivera

Universidad Autónoma de Coabuila, México

samanthaq.rivera@gmail.com

iD http://orcid.org/0000-0002-1332-8000
DOI: https://doi.org/10.15517/revedu.v44i1.35282

Redalyc: http://www.redalyc.org/articulo.oa?id=44060092014 
presenta un nivel estadísticamente mayor en su interés, percepción y expectativas de autoeficacia hacia el estudio de la matemática. Por otra parte, el alumnado de bachillerato mixto presenta mayores puntajes en el criterio relacionado con el coste de estudiar matemáticas.

Palabras ClaVe: Motivación, Educación a distancia, Educación postsecundaria, Modelo educacional.

\section{Abstract:}

The goal of this study is to statistically compare five aspects related of student motivation towards Mathematics among high school students involved in two types of learning programs - blended learning and face-to-face instruction. The study followed a quantitative methodology and selected a sample of 186 students enrolled in two types of public high school programs in Northern Mexico who were asked to fill out a questionnaire about their motivation towards Mathematics. The survey revealed their level of interest, importance, use, associated costs in studying Mathematics and self-efficacy expectations. According to the results, high school students in face-to-face learning programs had a statistically higher level of interest, perception and self-efficacy expectations about Mathematics. High School students enrolled in the blended learning modality had a higher score when answering the survey question about the financial cost associated with studying Mathematics.

KEYWORDS: Motivation, Distance Education, Distance-learning, Post-secondar, Education, Educational Model.

\section{INTRODUCCIÓN}

A lo largo de la historia, el principal objetivo de la educación ha sido brindar oportunidades al estudiantado para desarrollar su aprendizaje de la mejor manera posible en los distintos niveles educativos en donde se encuentren. En México, de acuerdo con el Artículo $3^{\circ}$ constitucional, el Estado tiene obligación de brindar oportunidades educativas a la niñez y la juventud del país desde el nivel preescolar hasta el bachillerato o nivel medio superior.

En aras de cumplir con esta responsabilidad y atender a la totalidad de estudiantes mexicanos, el Sistema Educativo ha diversificado las modalidades en las que se brinda la educación. La inclusión de modalidades adicionales a la tradicional presencial, ha sido posible debido a la evolución vertiginosa de las Nuevas Tecnologías de la Información y la Comunicación (TIC). El acceso a tecnología ha permitido diversificar las maneras de hacer llegar el conocimiento a quienes no pueden acceder a una escuela de manera presencial ya sea debido a barreras económicas o sociales (García, 2001). Específicamente en el nivel bachillerato, desde hace más de una década se han iniciado programas educativos en modalidades alternativas a la presencial, como la educación a distancia (e-learning), educación mixta (b-learning) y educación guiada por medios móviles (m-learning).

Sin embargo, no importando el tipo de modalidad educativa, todas buscan una educación integral para la formación de una ciudadanía competente, lo que incluye como preponderante el aprendizaje de las matemáticas y su aplicación a la vida cotidiana. Los programas de educación preparatoria (previa a la universidad) describen al aprendizaje de la matemática como una asignatura donde se busca que el alumnado formule y valide conjeturas, se planteen preguntas, usen procedimientos propios y logren comunicar, analizar e interpretar sus ideas y procedimientos de resolución (Quiroz, 2015).

Para el logro de tales objetivos, un aspecto muy importante a considerar, es la promoción y cuidado de la motivación de estudiantes. De acuerdo con Font (1994), la actitud hacia las matemáticas es influida por el patrón motivacional, el cual puede ser positivo o negativo. Si el patrón es positivo, el estudiantado, frente a una dificultad reacciona analizándola, buscará una nueva estrategia, preguntando a su docente. Si, por el contrario, presentan un patrón motivacional negativo, frente a una dificultad, aumenta su ansiedad y su angustia pensando que la causa de la dificultad es su incapacidad y, por tanto, adoptará una actitud defensiva, como, por ejemplo: no hacer nada, no preguntar, o intentar copiar respuestas (Font, 1994).

Por lo anterior, la presente investigación busca estudiar la forma en que la motivación hacia las matemáticas puede ser influida por la modalidad educativa en la que el alumnado se desenvuelve. Específicamente el objetivo del presente artículo es elaborar una comparación estadística de cinco aspectos de motivación entre 
estudiantes de bachillerato que cursan la modalidad presencial y la modalidad mixta. La pregunta que guía la investigación es: ¿Existen diferencias significativas en la motivación hacia las matemáticas entre estudiantes de bachillerato inscritos en modalidad presencial y aquellos inscritos en una modalidad mixta?

Se presentan a continuación los elementos conceptuales que fundamentaron la investigación, así como los aspectos relacionados con el contexto donde fue ejecutado el estudio. Posteriormente se describen las herramientas metodológicas que guiaron la recolección y análisis de datos, así como los resultados obtenidos que dan respuesta a la pregunta de investigación.

\section{Antecedentes PRÁCticos}

La modalidad educativa tradicional está caracterizada por el factor presencial, donde estudiantes asisten a clases personalmente y sus clases se desenvuelven de cara al profesorado, el cual guía las actividades de enseñanza. Diversos factores como el acceso a la información vía internet, la necesidad de horarios libres de estudio para el alumnado, entre otros, han hecho necesario el replanteamiento y cambio de las modalidades educativas. Según Rosales, Gómez, Durán, Salinas y Saldaña (2008) una razón importante que ha impulsado la inclusión de modalidades educativas alternas a la presencial es la rápida caducidad de los conocimientos, debido a la rapidez con que nueva información es producida. Para estos autores, las Tecnologías de la Información y la Comunicación (TIC), han permitido crear espacios donde se pueda aprender, aun sin coincidir en espacio ni en tiempo.

Ahora bien, la información y la tecnología usualmente avanzan a una velocidad superior a la metodología de la enseñanza. En la actualidad han proliferado las variedades y combinaciones de métodos de enseñanzaaprendizaje apoyadas en la nueva tecnología de informática, específicamente las modalidades de cursos pueden resumirse en: presencial o tradicional, haciendo uso de la World Wide Web (www); mixta (modalidad híbrida) presencial/en línea, y completamente en línea. Todas ellas están incluidas en lo que en la actualidad se ha denominado educación en línea, educación electrónica o educación virtual a ritmo propio (Rosales et al. 2008).

En México, la Secretaría de Educación Pública [SEP] a través de la Subsecretaría de Educación Media Superior, reconoce siete modalidades educativas en el bachillerato: Presencial, Intensiva, Virtual, Autoplaneada, Mixta, Certificación por Exámenes Parciales y Certificación por Examen (SEP, 2008).

La inclusión de modalidades alternas a la tradicional ha provocado un interés creciente de la investigación en materia de educación, por la realización de estudios comparativos entre estudiantes que cursan alguna de este tipo de educación. Específicamente han preponderado estudios relativos a la comparación entre educación tradicional presencial con modalidades híbridas o abiertas. Un ejemplo claro es la investigación de Rosales et al. (2008) quienes compararon el rendimiento académico en matemáticas de tres grupos de estudiantes, el primero y segundo en una modalidad presencial y el tercero en una modalidad híbrida. En los resultados presentados se evidenció que los tres grupos tuvieron mejoría en el rendimiento académico, sin embargo, fue mayor la mejora en el grupo de modalidad híbrida.

\section{REFERENTES TEÓRICOS - CONCEPTUALES}

Definir a la motivación se ha convertido en un desafío por los cambios progresivos de la psicología educativa, así como por las diferentes acepciones reconocidas por investigaciones que estudian la didáctica de diferentes disciplinas, entre ellas la didáctica de la matemática. Sin embargo, existe un consenso en la importancia de su estudio debido a la influencia que tiene en el aprendizaje de estudiantes. Además, es reconocido que la ausencia de motivación es una de las principales causas de fracaso escolar, por lo que su promoción es un objetivo del profesorado para el éxito de su enseñanza (Polanco, 2005). Así, de acuerdo con Sandoval et al. 
(2018), la motivación es un componente sumamente importante pues influye en la adaptación y el logro de diversos aprendizajes estudiantiles.

La motivación puede ser entendida como el proceso donde se despierta el interés y la atención de estudiantes por los valores contenidos en la misma asignatura, promoviendo el interés de aprenderla, el gusto por estudiarla y la satisfacción de cumplir las tareas que la materia les exige (Alves, 1963). De acuerdo con Campanario (2004) motivar supone apoyar a tener una predisposición a participar de manera activa en los trabajos del aula.

En la presente investigación se concuerda con la definición brindada por Santos citado por Polanco (2005), quien afirma que motivación se refiere "al grado en que los alumnos se esfuerzan para conseguir aquellas metas académicas que perciben como útiles y significativas" (p. 2). Más específicamente, el estudio refiere a metas académicas propias de la asignatura de matemáticas.

Las matemáticas suelen ser consideradas una asignatura de gran grado de complejidad, por lo que los estudios sobre cómo elevar su motivación han sido implementados en distintos niveles educativos (Alsina y Domingo, 2007). Ahora bien, los análisis efectuados en dichos estudios han sido enfocados a la relación de la motivación con diversas variables como el aprendizaje, la enseñanza y la evaluación de contenidos matemáticos (Orozco y Díaz, 2009, p.630).

Uno de los niveles donde más se ha enfocado el estudio de la motivación en matemáticas ha sido la escuela secundaria. El estudio de Cerezo y Casanova (2004) examina las diferencias de género existentes en distintas variables cognitivo-motivacionales y el rendimiento alcanzado en las asignaturas de lenguaje y matemáticas. Los resultados indican que estudiantes de sexo masculino presentan una mayor motivación extrínseca respecto a estudiantes de sexo femenino, sin embargo, no se presentan diferencias entre ellos cuando se trata de motivación intrínseca. Por otra parte, los estudios de Flores y Gómez (2010) y Gasco y Villarroel (2014) analizaron la relación de la motivación con el rendimiento académico, el grado escolar y el sexo. Los resultados de estos estudios muestran que la motivación aumenta cuando pasan del $2^{\circ}$ al $3^{\circ}$ curso de secundaria, además de una mayor autoeficacia de estudiantes de sexo masculino, aunque muy débil.

En el nivel bachillerato, investigaciones como la de Maseda (2011) han mostrado que la motivación hacia las matemáticas puede favorecerse si se utilizan metodologías activas en las cuales el alumnado descubra que las matemáticas son una asignatura entretenida, interesante y que se utiliza continuamente en la vida diaria. El estudio de las aplicaciones en matemáticas también ve favorecida la motivación en educación superior puesto que, de acuerdo con Polanco (2005), la verdadera motivación universitaria, proviene de encontrar una relación del estudio de las matemáticas hacia la carrera escogida, pues esto le ofrece un escalón más en la meta hacia su logro académico.

En el nivel superior, específicamente con estudiantes de Economía, Rojas, Escalera, Moreno y García (2017) muestran como la motivación y el gusto hacia la disciplina, la confianza en la habilidad para las matemáticas, la utilidad que vea cada estudiante en esta disciplina, así como el sentimiento de miedo, son factores que explican la actitud hacia las matemáticas.

Para mediar la motivación, algunas investigaciones han elaborado instrumentos que estimen las atribuciones al logro en matemáticas. Una de ellas es la propuesta de Orozco y Díaz (2009) quienes utilizan la Escala Atribucional de Motivación al Logro en Matemáticas [EAML-M] la cual incluye las seis causas más frecuentes atribuidas por parte del estudiantado que originan sus calificaciones en matemáticas: esfuerzo, interés, tarea, capacidad, exámenes y profesorado.

En la presente investigación se tomará en cuenta el cuestionario de motivación hacia las matemáticas guiada por la Teoría de expectativa valor, que fue ajustado a una escala Likert (de 1 a 5) por Gasco y Villarroel (2014). El test está dividido en cinco apartados que comprenden la motivación:

a) Interés: El interés se refiere al gusto y disfrute al estudio de las matemáticas.

b) Importancia: La importancia se evalúa mediante el valor otorgado a ser bueno en las asignaturas de matemáticas. 
c) Utilidad: La utilidad tiene como objetivo medir el grado de uso que se considera tienen las matemáticas para el futuro.

d) Coste: El coste se refiere a las actividades que se deben dejar de hacer para aprender matemáticas.

e) Expectativas de autoeficacia: Las expectativas de autoeficacia están en relación con la confianza del alumnado de obtener buenas calificaciones en la asignatura de matemáticas.

Se resalta el hecho de que las investigaciones mostradas anteriormente están implementadas en escuelas de modalidad presencial. García (2001) reconoce la necesidad de promover más investigaciones que abonen a la comprensión de los fenómenos inherentes al proceso de enseñanza-aprendizaje en alumnos y alumnas de educación a distancia e híbrida.

\section{Procedimientos metodológicos}

La presente investigación está enmarcada en un paradigma positivista por lo cual se elige una metodología cuantitativa. Esto es porque se busca que el manejo de los datos se ejecute de manera objetiva pretendiendo analizar las diferencias entre ellos (Kerlinger y Lee, 2002).

El diseño de investigación es semiexperimental. La población se conforma por estudiantes de bachillerato que cursan la modalidad presencial o mixta inscritos a la asignatura de Matemáticas I de dos instituciones de Educación Media Superior en el noreste de México. Las características específicas de cada institución son las siguientes:

- Bachillerato A. Esta institución educativa imparte educación media superior en su modalidad escolarizada presencial (tradicional). El alumnado tiene la obligación de asistir a clases en un horario fijo establecido, cumplir con las normas dentro de la institución, así como la elaboración de tareas y actividades en horarios fuera del escolar.

- Bachillerato B. Esta institución ofrece varias modalidades entre ellas educación mixta (combinación de educación presencial y a distancia). Los alumnos y las alumnas tienen la posibilidad de combinar sus estudios de manera virtual, y asistir a un plantel que cuenta con la tecnología para tomar una la clase virtual sincrónica (El/la docente explica la clase mediante una videoconferencia en tiempo real). Gran porcentaje del alumnado combinan sus estudios con su trabajo gracias a la flexibilidad de horarios y formas de aprender.

La muestra estuvo conformada por todo el estudiantado que formaba parte de la población que contestó el cuestionario que les fue enviado. Así, si se cumplía con las características arriba mencionadas y si estaban registrados en las instituciones elegidas fueron invitados a participar. Sin embargo, no se recolectó el 100\% de respuestas debido a diversos factores, entre ellos: había estudiantes que estaban ya dados de baja, además había quienes no tuvieron asistencia los días en que se ejecutó la encuesta, y también quienes no aceptaron la invitación a participar.

En total se tuvo una participación del 71,6\% del total de estudiantes de la modalidad presencial y 66,7\% del total de la modalidad mixta. La muestra final estuvo compuesta por un total de 186 estudiantes de nivel bachillerato donde 86 son de la modalidad presencial y 100 de la modalidad mixta. La edad de la muestra oscila entre los 14 y 19 años de edad. De la muestra correspondiente a la modalidad presencial 26 (30,23\%) son hombres y $60(69,76 \%)$ son mujeres. Respecto a la muestra de la modalidad mixta estaba compuesta por 40 (40\%) hombres y 60 (60\%) mujeres. La Tabla 1 muestra las características descriptivas de la muestra seleccionada. 
TABLA 1

Total de estudiantes y porcentajes, respecto de la educación modalidad mixta y presencial con respecto al género

\begin{tabular}{llll}
\hline GénerołModalidad & \multicolumn{2}{c}{$\begin{array}{l}\text { Modalidad } \\
\text { Mixta }\end{array}$} & $\begin{array}{l}\text { Modalidad } \\
\text { Presencial }\end{array}$ \\
\hline Hombres & $\%$ & $40,00 \%$ & $30,23 \%$ \\
\cline { 2 - 4 } & $\mathrm{N}$ & 40 & 26 \\
\hline Mujeres & $\%$ & $60,00 \%$ & $69,77 \%$ \\
\cline { 2 - 4 } & $\mathrm{N}$ & 60 & 60 \\
\hline Total & $\%$ & $53,76 \%$ & $46,24 \%$ \\
\cline { 2 - 4 } & $\mathrm{N}$ & 100 & 86 \\
\hline
\end{tabular}

Fuente: Elaboración propia

Para la medición de la motivación se utilizó el Cuestionario de motivación original de Berger y Karabenick (2011) y adaptado por Gasco y Villarroel (2014). El instrumento tiene como objetivo valorar la motivación académica hacia la matemática relacionada con las percepciones del alumnado. Por cada ítem, se tenía la posibilidad de responder de entre cinco respuestas plasmadas a manera de escala Likert, el grado de acuerdo o desacuerdo correspondiente con el enunciado. La distribución de los ítems se muestra en la Tabla 2. 
Revista Educación, 2020, 44(1), Enero-Junio, ISSN: 0379-7082 / 2215-2644

TABLA 2

Distribución de ítems

\begin{tabular}{|c|c|c|}
\hline Categoría & Ítem & $\begin{array}{l}\text { Número } \\
\text { de } \\
\text { item }\end{array}$ \\
\hline \multirow[t]{3}{*}{ Interés } & $\begin{array}{l}\text { Me gustan las } \\
\text { matemáticas }\end{array}$ & R01 \\
\hline & $\begin{array}{l}\text { Disfruto al } \\
\text { estudiar } \\
\text { matemáticas }\end{array}$ & $\mathrm{RO} 2$ \\
\hline & $\begin{array}{l}\text { Las } \\
\text { matemáticas } \\
\text { son } \\
\text { emocionantes }\end{array}$ & $\mathrm{RO3}$ \\
\hline \multirow[t]{3}{*}{ Importancia } & $\begin{array}{l}\text { Es } \\
\text { importante } \\
\text { para mi ser } \\
\text { alguien que } \\
\text { sea bueno en } \\
\text { matemáticas }\end{array}$ & R04 \\
\hline & $\begin{array}{l}\text { Creo que ser } \\
\text { bueno en } \\
\text { matemáticas } \\
\text { es parte } \\
\text { importante } \\
\text { de mi } \\
\text { personalidad }\end{array}$ & R05 \\
\hline & $\begin{array}{l}\text { Es } \\
\text { importante } \\
\text { para mí ser } \\
\text { alguien que } \\
\text { puede } \\
\text { razonar } \\
\text { utilizando } \\
\text { fórmulas y } \\
\text { operaciones } \\
\text { matemáticas }\end{array}$ & R06 \\
\hline \multirow[t]{3}{*}{ Utilidad } & $\begin{array}{l}\text { Creo que las } \\
\text { matemáticas } \\
\text { pueden ser } \\
\text { útiles en el } \\
\text { futuro } \\
\text { porque me } \\
\text { pueden } \\
\text { ayudar }\end{array}$ & R07 \\
\hline & $\begin{array}{l}\text { Creo que ser } \\
\text { bueno en } \\
\text { matemáticas } \\
\text { puede ser útil } \\
\text { en el futuro }\end{array}$ & R08 \\
\hline & $\begin{array}{l}\text { Creo que ser } \\
\text { bueno en } \\
\text { matemáticas } \\
\text { puede ser útil } \\
\text { para } \\
\text { encontrar } \\
\text { trabajo o } \\
\text { para ir a la } \\
\text { universidad }\end{array}$ & R09 \\
\hline \multirow[t]{2}{*}{ Coste } & $\begin{array}{l}\text { Tengo que } \\
\text { dejar de } \\
\text { hacer } \\
\text { muchas } \\
\text { cosas para } \\
\text { aprender } \\
\text { bien } \\
\text { matemáticas }\end{array}$ & R10 \\
\hline & $\begin{array}{l}\text { Creo que el } \\
\text { éxito en } \\
\text { matemáticas } \\
\text { requiere } \\
\text { dejar otras } \\
\text { actividades } \\
\text { que me } \\
\text { gustan }\end{array}$ & R11 \\
\hline \multirow[t]{3}{*}{$\begin{array}{l}\text { Expectativa } \\
\text { de } \\
\text { autoeficacia }\end{array}$} & $\begin{array}{l}\text { Creo que } \\
\text { tendré una } \\
\text { excelente } \\
\text { calificación } \\
\text { en } \\
\text { matemáticas }\end{array}$ & R12 \\
\hline & $\begin{array}{l}\text { Estoy seguro } \\
\text { de que puedo } \\
\text { entender los } \\
\text { contenidos } \\
\text { más difíciles } \\
\text { en } \\
\text { matemáticas }\end{array}$ & R13 \\
\hline & $\begin{array}{l}\text { Tengo } \\
\text { confianza en } \\
\text { que puedo } \\
\text { aprender los } \\
\text { conceptos } \\
\text { básicos } \\
\text { enseñados en } \\
\text { matemáticas }\end{array}$ & R14 \\
\hline
\end{tabular}


Fuente: Elaboración propia

Los catorce ítems se transcribieron en un formato en línea para que pudiera ser respondido desde una computadora o dispositivo móvil. Se les pidió que estos fueran contestados en el plantel escolar durante el tiempo de la clase de matemáticas. El período de aplicación fue de aproximadamente una semana. Las respuestas fueron analizadas y se presentan en el siguiente apartado.

\section{ANÁLISIS Y DISCUSIÓN DE RESULTADOS}

Inicialmente se pretendió conocer si existen diferencias significativas entre las puntuaciones medias de cada una de las dos modalidades educativas. Para ello, en un primer momento se efectuó la prueba Kolmogorov Smirnov en cada uno de los ítems para evaluar la normalidad de los datos. De acuerdo con Canavos (1988) se recomienda aplicar esta prueba en muestras mayores a 50 para determinar su normalidad. Los resultados se muestran en la Tabla 3.

TABLA 3

Resultados de la prueba de normalidad Kolmogorov Smirnov

\begin{tabular}{lllll}
\hline $\begin{array}{l}\text { Número } \\
\text { de }\end{array}$ & \multicolumn{2}{l}{$\begin{array}{l}\text { Modalidad } \\
\text { Mixta }\end{array}$} & \multicolumn{3}{l}{$\begin{array}{l}\text { Modalidad } \\
\text { presencial }\end{array}$} \\
\cline { 2 - 5 } item & KS & p & KS & P \\
\hline R01 & 0.239 & $<0.010$ & 0.265 & $<0.010$ \\
\hline R02 & 0.231 & $<0.010$ & 0.252 & $<0.010$ \\
\hline R03 & 0.203 & $<0.010$ & 0.218 & $<0.010$ \\
\hline R04 & 0.295 & $<0.010$ & 0.294 & $<0.010$ \\
\hline R05 & 0.239 & $<0.010$ & 0.206 & $<0.010$ \\
\hline R06 & 0.317 & $<0.010$ & 0.243 & $<0.010$ \\
\hline R07 & 0.274 & $<0.010$ & 0.333 & $<0.010$ \\
\hline R08 & 0.300 & $<0.010$ & 0.326 & $<0.010$ \\
\hline R09 & 0.253 & $<0.010$ & 0.267 & $<0.010$ \\
\hline R10 & 0.200 & $<0.010$ & 0.233 & $<0.010$ \\
\hline R11 & 0.205 & $<0.010$ & 0.244 & $<0.010$ \\
\hline R12 & 0.319 & $<0.010$ & 0.347 & $<0.010$ \\
\hline R13 & 0.276 & $<0.010$ & 0.342 & $<0.010$ \\
\hline R14 & 0.358 & $<0.010$ & 0.304 & $<0.010$ \\
\hline
\end{tabular}

Fuente: Elaboración propia

Siguiendo el criterio de la prueba de Kolmogorov Smirnov las respuestas de los 14 ítems tanto de la modalidad presencial como de la modalidad mixta presentan valores p menores a 0.01 por lo cual se puede concluir que las distribuciones no son normales o no presentan una homocedasticidad. Por lo anterior se decidió elegir pruebas no paramétricas para los siguientes análisis, específicamente la prueba Mann-Whitney la cual muestra si existen diferencias significativas entre dos muestras aleatorias que provienen de poblaciones cuyas distribuciones difieren de la tendencia central (Canavos, 1988; Anderson, Sweeney, Williams, Camm y Martin, 2011). Sus supuestos son los siguientes:

Las poblaciones son idénticas

Las poblaciones no son idénticas

En la Tabla 4 se presentan los análisis para las muestras de las dos modalidades. 
TABLA 4

Diferencia de medias en las dos modalidades educativas. Prueba U de Mann-Whitney

\begin{tabular}{|c|c|c|c|c|c|c|c|c|}
\hline \multirow{2}{*}{$\begin{array}{l}\text { Número } \\
\text { de } \\
\text { item }\end{array}$} & \multicolumn{3}{|c|}{ Modalidad Mixta } & \multicolumn{3}{|c|}{$\begin{array}{l}\text { Modalidad } \\
\text { Presencial }\end{array}$} & \multirow{2}{*}{$\begin{array}{l}\text { U de } \\
\text { Mann- } \\
\text { Whitney }\end{array}$} & \multirow[t]{2}{*}{$\mathrm{p}$} \\
\hline & $\mathrm{N}$ & Media & DT & $\mathrm{N}$ & Media & DT & & \\
\hline R01 & 100 & 3.08 & 1.09 & 86 & 3.67 & 0.79 & 9394.0 & 0.0001 \\
\hline R02 & 100 & 3.12 & 1.01 & 86 & 3.62 & 0.87 & 9228.0 & 0.0006 \\
\hline RO3 & 100 & 3.10 & 0.97 & 86 & 3.38 & 0.92 & 8768.0 & 0.0359 \\
\hline R04 & 100 & 3.88 & 1.06 & 86 & 4.29 & 0.63 & 8893.5 & 0.0112 \\
\hline R05 & 100 & 3.41 & 1.13 & 86 & 3.53 & 0.98 & 8214.0 & 0.6236 \\
\hline R06 & 100 & 3.76 & 0.98 & 86 & 3.98 & 0.91 & 8529.5 & 0.1526 \\
\hline R07 & 100 & 4.04 & 0.99 & 86 & 4.51 & 0.65 & 9223.0 & 0.0004 \\
\hline R08 & 100 & 4.05 & 0.98 & 86 & 4.49 & 0.66 & 9180.5 & 0.0006 \\
\hline R09 & 100 & 3.82 & 1.14 & 86 & 4.30 & 0.78 & 9063.5 & 0.0029 \\
\hline R10 & 100 & 3.24 & 1.04 & 86 & 2.88 & 0.98 & 7102.0 & 0.0076 \\
\hline R11 & 100 & 2.92 & 1.03 & 86 & 2.34 & 1.01 & 6694.5 & 0.0001 \\
\hline R12 & 100 & 4.23 & 1.20 & 86 & 4.36 & 1.04 & 9152.0 & 0.5390 \\
\hline R13 & 100 & 4.31 & 0.84 & 86 & 4.48 & 0.78 & 8813.5 & 0.0990 \\
\hline R14 & 100 & 3.77 & 0.84 & 86 & 3.98 & 0.70 & 8875.0 & 0.1380 \\
\hline
\end{tabular}

Fuente: Elaboración propia

La comparación de las respuestas de los ítems R01, R02, R03, R04, R07, R08, R09, R10 y R11 reportan diferencias significativas con valores. Por otro lado, los ítems R05, R06, R12, R13 y R14 presentan valores por lo que indican que no existen diferencias significativas entre las respuestas de estudiantes de la modalidad mixta y estudiantes de la modalidad presencial.

Por lo anterior, se decidió plantear una nueva hipótesis para los ítems en donde se detectaron diferencias significativas, esto para detectar la modalidad educativa en la que se presentó mayor motivación.

Las nuevas hipótesis de investigación determinaban:

Las poblaciones son idénticas

La población 1 es mayor que la población 2

La Tabla 5 muestra los ítems que mostraron valores que indican que las respuestas del alumnado de la modalidad presencial fueron estadísticamente mayores que el estudiantado de la modalidad mixta.

TABLA 5.

Población 1 (Modalidad presencial) > Población 2 (Modalidad mixta)

\begin{tabular}{lll}
\hline $\begin{array}{l}\text { Número } \\
\text { de } \\
\text { item }\end{array}$ & $\begin{array}{l}\text { U de } \\
\text { Mann-Whitney }\end{array}$ & $\mathrm{P}$ \\
\hline R01 & 9394.0 & 0.0000 \\
\hline R02 & 9228.0 & 0.0030 \\
\hline R03 & 8768.0 & 0.0179 \\
\hline R04 & 8893.5 & 0.0056 \\
\hline R07 & 9251.5 & 0.0010 \\
\hline R08 & 9180.5 & 0.0030 \\
\hline R09 & 9063.5 & 0.0014 \\
\hline R13 & 8575.5 & 0.0495 \\
\hline & & \\
\hline
\end{tabular}

Fuente: Elaboración propia

En la Tabla 6 se muestran los ítems faltantes donde se buscó conocer la hipótesis contraria a la referida en la Tabla 5, es decir, buscar si la población 2 era mayor que la población 1 :

Las poblaciones son idénticas 
La población 2 es mayor que la población 1

TABLA 6

Población 2 (Modalidad Mixta) > Población 1 (Modalidad Presencial)

\begin{tabular}{lll}
\hline $\begin{array}{l}\text { Número } \\
\text { de } \\
\text { item }\end{array}$ & $\begin{array}{l}\text { Mann-Whitney } \\
\text { R10 }\end{array}$ & P \\
\hline R11 & 10289.0 & 0.0038 \\
\hline
\end{tabular}

Fuente: Elaboración propia

Los resultados de la prueba U de Mann-Whitney aplicados a los ítems R10 y R11 se muestran en la Tabla 6. Estos muestran que, en los ítems referidos, las respuestas de los alumnos y las alumnas de la modalidad mixta fueron mayores estadísticamente que la modalidad presencial.

La discusión de resultados se complementará profundizando en cada uno de los cinco apartados que comprenden la motivación de acuerdo al test utilizado:

a) Interés

Fueron tres los ítems que midieron el interés del estudiantado hacia las matemáticas:

- Me gustan las matemáticas

- Disfruto al estudiar matemáticas

- Las matemáticas son emocionantes

Se detectó que el alumnado de la modalidad presencial, muestran mayor interés en cuanto al gusto y disfrute de las matemáticas. De acuerdo con González (2005) el interés hacia las matemáticas está relacionado con la percepción sobre la dificultad de la asignatura. Una razón que podría explicar lo anterior la establecen Atweh, Forgasz y Nebres (2001) quienes argumentan que las aulas donde se utilizan libros de texto que muestran el placer producido al resolver problemas matemáticos, así como el cuerpo docente que promueve este gusto, provoca un mayor disfrute y emoción hacia la asignatura.

b) Importancia

El primer ítem relacionado con la importancia hacia el estudio de las matemáticas fue Es importante para mi ser alguien que sea bueno en matemáticas. Las pruebas efectuadas a las respuestas mostraron diferencias significativas entre ambas modalidades. Específicamente se presentó un valor mayor en las respuestas de la modalidad presencial respecto la modalidad mixta.

Los otros dos ítems R05 Creo que ser bueno en matemáticas es parte importante de mi personalidad y R06 Es importante para mi ser alguien que puede razonar utilizando fórmulas y operaciones matemáticas no presentaron diferencias significativas entre las dos modalidades educativas. Por lo anterior es posible concluir que ambos grupos valoran de igual manera la importancia de las matemáticas en su formación académica.

Ahora bien, se puede señalar igualmente que el alumnado de la modalidad presencial considera más valioso o de mayor importancia el ser alguien bueno en matemáticas respecto al alumnado de la modalidad mixta. De acuerdo con Carrillo, Padilla, Rosero y Villagómez (2009) los y las docentes presenciales apoyan el sentimiento de competencia y este puede ser un factor clave para elevar la motivación. Ello debido a que se logra pensar que se puede aprender y que hay sentido el ejecutar un esfuerzo para conseguir sus metas.

c) Utilidad 
La motivación hacia las matemáticas puede estar influenciada por la utilidad de la misma en un grado escolar mayor al cual se encuentra actualmente el estudiante, o en un contexto diferente al escolar. Al analizar este aspecto de la motivación, se encontró que, en los tres ítems referidos a la utilidad, el alumnado de la modalidad presencial tuvo un puntaje estadísticamente mayor que el alumnado de la educación mixta.

Los ítems estribaban en indicar si las matemáticas podían ser útiles en el futuro, así como si el ser bueno en matemáticas podría resultar útil en el futuro para encontrar empleo o en la universidad. Los resultados evidencian que en la educación mixta no es tan fácil hacer ver la utilidad de las matemáticas, así como el aprendizaje de habilidades y conceptos matemáticos para un buen funcionamiento de la sociedad (Mato y de la Torre, 2009).

d) Coste

El coste se define como la renuncia que supone el aprendizaje de las matemáticas de cara a desarrollar otras actividades. El estudio del coste no ha sido desarrollado de manera amplia en la literatura, sin embargo, se ha demostrado que la percepción del coste en estudiantes de diferentes niveles educativos no evoluciona, sino que se mantiene constante (Gasco y Villarroel, 2014). En la presente investigación, los resultados muestran que el alumnado de la modalidad mixta le otorga valores más altos al coste que el estudiantado de la modalidad presencial. Específicamente, la muestra correspondiente concuerda en que tienen que dejar de hacer muchas cosas para aprender bien matemáticas y que el éxito en matemáticas requiere dejar otras actividades que les gustan.

Estos resultados se relacionan con lo establecido por García (2001) quien describe a las modalidades alternas a la presencial, especialmente a modalidades que reúnen aprendizaje presencial y a distancia, como dedicadas a un público específico que tiene responsabilidades laborales además de las escolares. Es decir, al ser personas que trabajan en su mayoría, los alumnos y las alumnas indican que aprender matemáticas les demanda un mayor tiempo del que dedican a otras actividades.

e) Expectativas de Autoeficacia

La expectativa de autoeficacia hacia las matemáticas mide si los y las estudiantes se consideran capaces de obtener resultados satisfactorios en esta asignatura. Por ello, es un factor que tiene impacto en la motivación hacia las matemáticas (Lamas, 2008; Steinmann, Bosch y Aiassa, 2013).

Para medir las expectativas de autoeficacia se destinaron tres ítems específicos:

- Creo que tendré una excelente calificación en matemáticas

- Estoy seguro de que puedo entender los contenidos más difíciles en matemáticas

- Tengo confianza en que puedo aprender los conceptos básicos enseñados en matemática

Los resultados muestran que la muestra correspondiente a la modalidad presencial mostró mayor expectativa de autoeficacia que los de la otra modalidad, referente al entendimiento de los contenidos más difíciles en matemáticas. Por otro lado, las respuestas de los ítems 12 y 14 no tienen diferencias significativas.

El R13 la media de los alumnos y las alumnas de los bachilleratos mixto y presencial fue 4,31 y 4,48 respectivamente, por lo cual se puede observar que en ambas modalidades se tienen niveles altos de creencia de que obtendrán buenas calificaciones en matemáticas. Las medias del R14 muestran medias un poco menos altas en ambos grupos (3,77 y 3,98), sin embargo, reflejan la confianza de ambos grupos de aprender los conceptos enseñados en la matemática.

\section{Conclusiones}

Promover la motivación en el aprendizaje de las matemáticas es indispensable para lograr que cada estudiante se comprometa con su propio proceso de aprendizaje. En la presente investigación se mostraron cinco 
aspectos que deben ser considerados cuando se mide el nivel de motivación hacia las matemáticas del alumnado: el interés, la importancia, la utilidad, el coste y las expectativas de autoeficacia.

La conclusión más representativa del estudio estriba en la evidencia de diferencias significativas respecto a la motivación hacia las matemáticas de estudiantes de bachillerato, de las modalidades mixta y presencial. Se mostró que estudiantes de la modalidad presencial mostraron mayores niveles de interés hacia el estudio de la matemática, y una mayor percepción de que esta asignatura será de utilidad para sus actividades futuras. Alguna de las razones de estos resultados puede deberse a que el alumnado del bachillerato presencial cuenta con más tiempo dedicado exclusivamente para el estudio de las asignaturas y la mayoría de ellos tiene planes de continuar sus estudios universitarios.

Por otro lado, el apartado de coste, tuvo mayores niveles en la modalidad mixta. Este resultado muestra que el estudiantado de la modalidad mixta reconoce que estudiar matemáticas tiene un costo relativo a tener menos tiempo de desarrollar otras actividades. Esto puede ser explicado porque la mayoría de ellos combinan su trabajo con los estudios. Además, el alumnado de este tipo de modalidad no tiene la ventaja de poder resolver sus dudas en matemáticas de manera inmediata por el hecho de la poca frecuencia de horas de asesoría a la semana.

Se evidencia que la promoción de una mayor motivación puede estar relacionado con el acompañamiento y la mediación del profesorado en todo momento. Sin embargo, el estudio concuerda con Urquijo (2009) y se considera que en ambas modalidades puede lograrse lo anterior si se diseñan correctamente entornos de aprendizaje que permitan a los y las docentes guiar su proceso de enseñanza. En la modalidad mixta esto implica un proceso integral y armonizado de todos los elementos los cuales conforman el entorno virtual, así como programas, contenidos y sujetos que intervienen. Las modalidades mixtas requieren, por tanto, elegir plataformas que fomenten la comunicación abierta y la interactividad (Vecchini, Vega, Torres y Irizarry, 2016).

El presente artículo considera importante continuar con estudios que busquen comprender los procesos de enseñanza y aprendizaje de las matemáticas en modalidades alternativas a la presencial. Específicamente, indagar más profundamente sobre alternativas donde se analicen los factores que se relacionan con el incremento de la motivación en diversos niveles educativos. Además de esto, la comunicación de estos resultados hacia las escuelas y docentes podría apoyar la comprensión de estos hacia los problemas de motivación, propiciando el diseño de estrategias de mejora en las diferentes modalidades educativas.

\section{REFERENCIAS}

Alsina, Á y Domingo, M. (2007). Cómo aumentar la motivación para aprender matemáticas. Suma, 56(1), 23-31.

Alves, L. (1963). Compendio de Didáctica General. Madrid, España: Editorial Kapelusz.

Anderson, D., Sweeney, D., Williams, T., Camm, J. y Martin, K. (2011). Métodos cuantitativospara los negocios. Ciudad de México, México: Cengage Learning.

Atweh, B., Forgasz, H. y Nebres, B. (2001). Sociocultural Research on Mathematics Education. An International Perspective. Nueva Jersey, U.S.A.: Lawrence Erlbaum Associates.

Berger, J.y Karabenick, S. (2011). Motivation and students' use of learning strategies: Evidence of unidirectional effects in mathematics classrooms. Learning and instruction, 21(3), 416-428.

Campanario, J. (2004). Algunas posibilidades del artículo de investigación como recurso didáctico orientado a cuestionar ideas inadecuadas sobre la ciencia. Revista Enseñanza de las ciencias, 2(22), 365-378.

Canavos, G. (1988). Probabilidad y estadística: aplicaciones y métodos. Ciudad de México, México: McGraw-Hill.

Carrillo, M., Padilla, J., Rosero, T. y Villagómez, M. (2009). La motivación y el aprendizaje. Revista de Educación, 2(4), 20-32.

Cerezo, M. y Casanova, P. (2004). Diferencias de género en la motivación académica de los alumnos de Educación Secundaria Obligatoria. Electronic journal of research in educational psychology, 1(2), 97-112. 
García L. (2001). La educación a distancia: De la teoría a la práctica. Barcelona, España: Editorial Ariel Educación.

Gasco, J.y Villarroel, J. (2014). The Motivation of Secondary School Students in Mathematical Word Problem Solving. Electronic Journal of Research in Educational Psychology, 12(1), 83-106.

González, R. (2005). Un modelo explicativo del interés hacia las matemáticas de las y los estudiantes de secundaria. Educación Matemática, 17(1), 107-128.

Flores, R. y Gómez, J. (2010). Un estudio sobre la motivación hacia la escuela secundaria en estudiantes mexicanos. Revista electrónica de investigación educativa, 12(1), 1-18.

Font, V. (1994). Motivación y dificultades de aprendizaje en matemáticas. Suma, 17(1), 10-16.

Kerlinger, F. y Lee, H. (2002). Investigación del comportamiento: métodos de investigación y ciencias sociales. Ciudad de México, México: Mc Graw Hill.

Lamas, H. (2008) Aprendizaje autorregulado, motivación y rendimiento académico. Liberabit, 14(14), 15-20.

Maseda, M. (2011). Estudio bibliográfico de la motivación en el aprendizaje de las matemáticas y propuesta de talleres aplicados a la vida real. (Tesis de maestría). Universidad Internacional de la Rioja, México, CDMX.

Mato, M. y de la Torre, E. (2009). Evaluación de las actitudes hacia las matemáticas y el rendimiento académico. En M.J. González, M.T. González y J. Murillo (Ed.), Investigación en Educación Matemática XIII (pp. 285-300). Santander: SEIEM.

Orozco, C. y Díaz, M. (2009). Atribuciones de la motivación al logro y sus implicaciones en la formación del pensamiento lógico-matemático en la Universidad. Interciencia, 34(9), 630-636.

Polanco, A. (2005). La motivación en los estudiantes universitarios. Revista Actualidades Investigativas en Educación, $5(2), 1-13$.

Quiroz, S. (2015). Concepciones de Modelación matemática de docentes en formación de educación primaria (Tesis de Doctorado) Escuela de Educación, Humanidades y Ciencias Sociales del Tecnológico de Monterrey, Monterrey, México

Rojas, C., Escalera, M., Moreno, E., García, A. (2017). Motivación, ansiedad, confianza, agrado y utilidad. Los factores que explican la actitud hacia las matemáticas en los estudiantes de Economía. International Journal of development and Educational Psychology, 2(1), 527-539.

Rosales, S., Gómez, V., Durán, S., Salinas, M. y Saldaña, S. (2008). Modalidad híbrida y presencial. Comparación de dos modalidades educativas. Revista de la Educación Superior, 37(148), 23-29.

Sandoval, M., Mayorga, C., Elgueta, H., Soto, A., Viveros, J., y Riquelme, S. (2018). Compromiso y motivación escolar: una discusión conceptual. Revista Educación, 42(1), 119-146.

Secretaria de Educación Pública [SEP]. (2008). Define SEP siete modalidades para regular escuelas de Educación Media Superior y elevar la calidad. Recuperado de https://bit.ly/31Nf34f

Steinmann, A., Bosch, B. y Aiassa, D. (2013). Motivación y expectativas de los estudiantes por aprender ciencias en la Universidad: un estudio exploratorio. Revista mexicana de investigación educativa, 18(57), 585-598.

Urquijo, M. (2009). Competencias que desarrolla el estudiante universitario que participa en programas en modalidades alternativas. (El caso del Centro de Educación a Distancia, Universidad La Salle, México). Revista del Centro de Investigación Universidad La Salle, 8(32), 67-80.

Vecchini, R., Vega, A., Torres, Y. y Irizarry, E. (2016). Hybrid Courses: From another perspective. Tecné, 8(1), 1-12.

CC BY-NC-ND 